\title{
Intratumoural budding (ITB) in preoperative biopsies predicts the presence of lymph node and distant metastases in colon and rectal cancer patients
}

\author{
I Zlobec ${ }^{\star 1}$, M Hädrich², H Dawson ${ }^{1,3}$, V H Koelzer ${ }^{1,3}$, M Borner $^{4}$, M Mallaev $^{1,2}$, B Schnüriger ${ }^{2}$, D Inderbitzin² \\ and A Lugli ${ }^{1,3}$ \\ ${ }^{1}$ Translational Research Unit (TRU), Institute of Pathology, University of Bern, Bern, Switzerland; ${ }^{2}$ Department of Visceral Surgery \\ and Medicine, Bern University Hospital, Bern, Switzerland; ${ }^{3}$ Division of Clinical Pathology, Institute of Pathology, University of \\ Bern, Bern, Switzerland and ${ }^{4}$ Department of Oncology, Hospital Centre Biel, Biel, Switzerland
}

Background: In colorectal cancer (CRC), tumour budding at the invasion front is associated with lymph node (LN) and distant metastasis. Interestingly, tumour budding can also be detected in biopsies (intratumoural budding; ITB) and may have similar clinical importance. Here we investigate whether ITB in preoperative CRC biopsies can be translated into daily diagnostic practice.

Methods: Preoperative biopsies from 133 CRC patients (no neoadjuvant therapy) underwent immunohistochemistry for pancytokeratin marker AE1/AE3. Across all biopsies for each patient, the densest region of buds at $\times 40$ (high-power field; HPF) was identified and buds were counted.

Results: A greater number of tumour buds in the biopsy was associated with pT stage $(P=0.0143), L N$ metastasis $(P=0.0007)$, lymphatic $(P=0.0065)$ and venous vessel invasion $(P=0.0318)$ and distant metastasis $(\mathrm{cM} 1)(P=0.0013)$. Using logistic regression, a 'scale' was developed to estimate the probability of LN and distant metastasis using the number of tumour buds (e.g. 10 buds per HPF: 64\% chance of LN metastasis; 30 buds per HPF: $86 \%$ chance). Inter-observer agreement for ITB was excellent (intraclass correlation coefficient: 0.813 ).

Conclusion: Tumour budding can be assessed in the preoperative biopsy of CRC patients. It is practical, reproducible and predictive of LN and distant metastasis. Intratumoural budding qualifies for further investigation in the prospective setting.

The preoperative management of colorectal cancer (CRC) is based on an interdisciplinary collaboration between oncologists, gastroenterologists, surgeons, radiologists and pathologists. Indeed, rectal cancer patients with an advanced T stage (cT3 or cT4) and/or presence of lymph node (LN) metastases $(\mathrm{N}+)$ are typically selected for neoadjuvant therapy (Schmoll et al, 2012). In the preoperative setting, the pathologist's role is limited to the confirmation of a diagnosis of malignancy from the preoperative biopsy as the neoplastic nature of the lesion is already highly suspected from the clinical point of view. Although many histomorphological parameters such as tumour grade, lymphatic and vascular invasion, tumour border configuration, perineural infiltration, intra- and peritumoural inflammation and histological subtype should be reported in the daily practice (Bosman et al, 2010), their use is limited to the resection; consequently, these features do not influence the preoperative management of the patient as they cannot be applied to biopsies. 
Over the last years, a novel and promising histomorphological parameter, namely 'tumour budding' has been reported in many studies (Okuyama et al, 2002; Ueno et al, 2002; Okuyama et al, 2003; Tanaka et al, 2003; Ueno et al, 2004; Nakamura et al, 2005; Park et al, 2005; Prall et al, 2005; Wang et al, 2009). Tumour budding is defined as the presence of single tumour cells or small tumour cell clusters of up to five cells at the invasive front of CRC. The presence of tumour budding is associated with LN and distant metastases as well as worse survival, independently of the applied scoring system (Okuyama et al, 2002; Ueno et al, 2002, 2004; Okuyama et al, 2003; Tanaka et al, 2003; Nakamura et al, 2005; Park et al, 2005; Prall et al, 2005; Wang et al, 2009). In 1989, Morodomi et al (1989) described the presence of budding in biopsies from rectal cancer patients and its strong association with lymphatic invasion and LN metastasis. In 2011, influenced by the promising prognostic parameters intratumoural and peritumoural lymphocytic infiltration in CRC, we systematically analysed wholetissue sections from the surgical resection and the presence of tumour budding within the whole tumour mass and introduced the terms intratumoural (ITB) and peritumoural (PTB) budding to characterise the location of tumour buds either within the tumour centre or at the invasion front of CRC, respectively (Lugli et al, 2011). The promising diagnostic power of ITB in preoperative biopsies of colon and rectal cancers was recently confirmed by two studies with 72 and 89 patients (Giger et al, 2012; Rogers et al, 2014).

The aim of the present study was to investigate on a cohort of colon and rectal cancer patients not receiving neoadjuvant therapy $(N=133)$ and with full histopathological data whether ITB in preoperative biopsies can be translated into daily diagnostic practice. To this end, we use well-established methods, namely immunohistochemistry (pan-cytokeratin marker) to optimally visualise tumour buds and a high-power field method of scoring, which is already used to count mitoses in breast cancer, sarcomas and gastrointestinal stromal tumours.

\section{MATERIALS AND METHODS}

Patients. All patients with primary CRC surgically treated between 2002 and 2011 at the University Hospital Insel, Bern, Switzerland were initially entered into this study. Three hundred and forty-six patients were identified. Of these, 185 had a matched preoperative diagnostic biopsy available. For these 185 cases, Hematoxylin and Eosin (H\&E) slides from surgical resections were reviewed by one experienced gastrointestinal pathologist $(\mathrm{AL})$ and two residents-in-training (HD and VK), blinded to findings from the preoperative biopsy. The following histopathological parameters were revised: TNM (7th edition), histological subtype, tumour grade, lymphatic invasion (L), venous invasion (V), number of tumour deposits, perineural invasion (Pn), the number of LNs harvested and regression grade according to the College of American Pathologists (CAP) for patients preoperatively treated with radio and/or chemotherapy. All cases were grossed according to the national guidelines on quality assurance provided by the Swiss Society of Pathology: All loco-regional LNs were macroscopically identified by careful and meticulous examination of the pericolonic fat. Lymph nodes with a diameter below $0.5 \mathrm{~cm}$ were embedded completely and examined on H\&E. Nodes with a diameter between 0.5 and $0.7 \mathrm{~cm}$ were bisected and nodes greater than $0.7 \mathrm{~cm}$ in diameter were sectioned at $0.2 \mathrm{~cm}$ intervals and embedded completely. Clinical data were obtained from patient records and included age at diagnosis, gender, tumour location, clinical TNM staging, neoadjuvant and adjuvant therapy information. Clinical staging was performed primarily by computer tomography (CT) (including PET-CT) followed by magnetic resonance imaging (MRI) and ultrasound. Thirty patients receiving neoadjuvant or preoperative therapy were excluded from further analysis. Histopathological re-review of the preoperative biopsies was also undertaken. In addition, information on the number of biopsies per patient and the number of biopsies containing tumour was determined.

Assay methods. All biopsies were fixed in 10\% buffered formalin, paraffin-embedded and archived at the Institute of Pathology, University of Bern, Switzerland. Tumour blocks were retrieved and sectioned at $4 \mu \mathrm{m}$. An immunohistochemistry double-stain for pan-cytokeratin marker AE1/AE3 (Dako, Glostrup, Denmark, mouse monoclonal, $11: 200$, enzyme pretreatment $5 \mathrm{~min}$; DAB chromogen;) and CD8 + (Dako C8/144B, 1:100, pretreatment with Tris buffer, at $95^{\circ}$ for $20 \mathrm{~min}$; AEC chromogen) using an automated Leica Bond III instrument and counterstained with Hematoxylin was performed. Pan-cytokeratin staining was used to evaluate tumour buds. This study was approved by the local ethics committee of the Insel Hospital (16 March 2012).

Assessment of tumour budding. Tumour budding was assessed using the following method. All biopsies from a single patient were scanned and those containing tumour were evaluated. One single tumour hot-spot containing the greatest density of tumour buds (Nikon Eclipse 50i microscope at $\times 40$ magnification, field area $0.1963 \mathrm{~mm}^{2}$ ) upon pan-cytokeratin AE1/AE3 staining was identified and the number of tumour buds was counted and recorded. Tumour buds were defined as single tumour cell of clusters of up to five cells or single tumour cells with a defined nucleus and diffuse cytoplasmic staining for AE1/AE3 (Figure 1). Tumour budding counts from two independent observers (AL and HD) were utilised to determine the inter-observer agreement of the scoring method.

Study design. The study design is outlined in Figure 2. Of the 346 patients initially identified as having surgical treatment for primary CRC from 2002 to 2011, 185 had matched biopsies. Of these, 23 patients were further excluded as they received a preoperative radio/or chemotherapy. Immunohistochemistry was performed on the remaining cases and were scored for tumour budding using the hot-spot method. Upon re-review of stained biopsies, 29 cases were further excluded since, due to sectioning of the biopsy, only few tumour cells remained for evaluation. The final number of

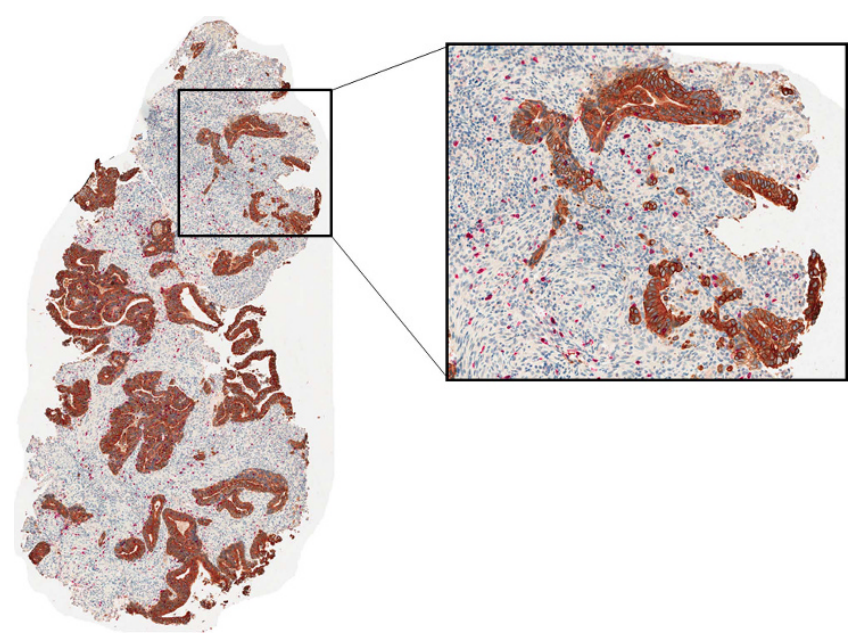

Figure 1. Assessment of intratumoural budding. Preoperative colonic biopsy stained for pan-cytokeratin (brown) to highlight single tumour buds. The densest area (hot-spot) of intratumoural budding is identified. At $\times 40$ magnification, the total number of tumour buds in one single HPF is counted. The full colour version of this figure is available at British Journal of Cancer online. 


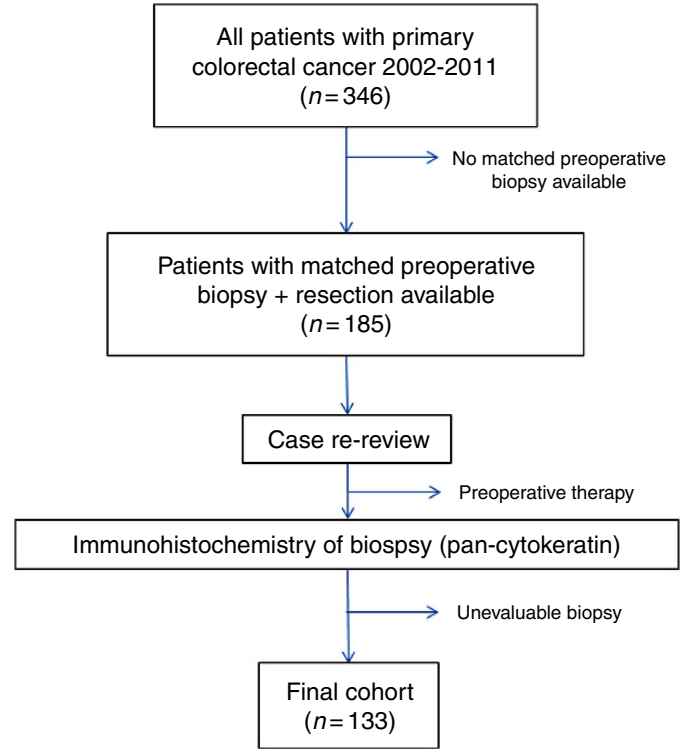

Figure 2. Study design. Out of an initial cohort of 346 patients, matched preoperative biopsies were available for 185. Cases were re-reviewed. Patients receiving preoperative therapy were further excluded. Biopsies underwent immunohistochemistry and the final number of evaluable cases was 133.

matched preoperative biopsies with tumour budding scores and completely re-reviewed surgical resections was 133 . Patient characteristics are listed in Table 1.

Statistics. The non-parametric Wilcoxon Rank Sum or KruskalWallis test was used to compare ranks of tumour budding scores for categorical variables. For age and total LNs collected, the estimate for tumour buds from the linear regression model was used. The inter-observer agreement was determined using the intraclass correlation coefficient (ICC), with values approaching 1.0 indicating improved agreement. Logistic regression analysis was performed to calculate the probability of LN metastasis and distant metastasis as a function of the number of tumour buds in the biopsy. Briefly, the probability $P=e^{\left(\beta_{\mathrm{o}}+\beta 1_{x}\right)} /\left(1-e^{\left(\beta_{\mathrm{O}}+\beta 1_{x}\right)}\right)$, where $\beta_{\mathrm{o}}$ and $\beta_{1}$ are estimates of the logistic equation and $\mathrm{x}$ is the number of tumour buds. In addition, odds ratios (ORs) and $95 \%$ confidence intervals (CI) were used to determine the effect of tumour budding on each significant variable. The area under the ROC curve (AUC) was used to determine the discriminatory ability of budding for the feature.

\section{RESULTS}

Tumour budding in the biopsy. An average of 12 tumour buds per hot-spot was found with a median of five buds. Ninety percent of all cases showed $<25$ buds per hot-spot. In more detail, 97 patients had between 0 and 10 buds, 19 had 11-20 buds, 7 had 21-30 buds and 6 had 31-50 buds per hot-spot. Only four cases had $>50$ buds per hot-spot and all four were of signet ring cell histology. The inter-observer assessment was performed on 133 cases. The ICC was 0.801 indicating excellent agreement between independent observers. All cases with $>50$ buds per hot-spot were removed and again assessed for agreement. The ICC was 0.72 indicating strong agreement.

Tumour budding in the biopsy and clinicopathological features. The association between a greater number of tumour buds in the biopsy and both clinical and histopathological features of the surgical resection is outlined in Table 2. A greater number of
Table 1. Characteristics of patients with preoperative biopsy and matched oncosurgical resection $(N=133)$

\begin{tabular}{|l|c|}
\hline \multicolumn{2}{|l|}{} \\
\hline Age (years) \\
\hline Median (min, max) & $72.2(30-91)$ \\
\hline Gender & \\
\hline Male & $79(59.4)$ \\
Female & $54(40.6)$
\end{tabular}

Histological subtype

\begin{tabular}{|l|r|}
\hline Non-mucinous & $107(81.1)$ \\
Mucinous & $25(18.9)$ \\
\hline
\end{tabular}

\section{Tumour location}

\begin{tabular}{|l|r|}
\hline Left & $42(31.8)$ \\
Rectum & $31(23.5)$ \\
Right & $59(44.7)$
\end{tabular}

\begin{tabular}{|l|l|}
\hline pT \\
\hline pT1 & $10(7.5)$ \\
pT2 & $22(16.5)$ \\
pT3 & $71(53.4)$ \\
pT4 & $30(22.6)$ \\
\hline pN & \\
\hline pN0 & $56(42.1)$ \\
pN1-2 & $77(57.9)$ \\
\hline
\end{tabular}

No. lymph nodes collected

\begin{tabular}{|l|l}
\hline Median (min, max) & $22(4-73)$ \\
\hline
\end{tabular}

Tumour deposits

\begin{tabular}{|c|r}
\hline 0 & $94(84.7)$ \\
\hline 1 & $12(10.8)$
\end{tabular}

$\begin{array}{ll}0 & 12(10.8)\end{array}$

$>1$

$5(3.5)$

\begin{tabular}{|l|l|}
\hline \multicolumn{2}{|l|}{ Metastasis } \\
\hline CM0 & $88(69.3)$ \\
CM1 & $39(30.7)$
\end{tabular}

Lymphatic invasion

\begin{tabular}{|l|r}
\hline LO & $30(25.9)$ \\
\hline
\end{tabular}

L1 $\quad 86(74.1)$

Venous invasion

\begin{tabular}{|l|r}
\hline V0 & $58(49.6)$ \\
V1-2 & $59(51.4)$
\end{tabular}

Perineural invasion $(\mathrm{Pn})$

\begin{tabular}{|r|r}
\hline 0 & $101(88.6)$ \\
1 & $13(11.4)$ \\
\hline
\end{tabular}

Tumour grade

\begin{tabular}{|l|r}
\hline G1-2 & $90(65.4)$ \\
G3 & $46(34.6)$
\end{tabular}

Adjuvant therapy

\begin{tabular}{|l|l|}
\hline None & $89(67.9)$ \\
Yes & $42(62.1)$ \\
\hline
\end{tabular}


Table 2. Association of budding in the preoperative biopsy (median number of buds) and clinicopathological features of the resected specimen in patients not treated with neoadjuvant therapy $(N=133)$

\begin{tabular}{|c|c|c|c|c|}
\hline & $\begin{array}{c}\text { ITB } \\
\text { median }\end{array}$ & $P$-value & OR $(95 \% \mathrm{Cl})$ & AUC \\
\hline \multicolumn{5}{|l|}{ Age (years) } \\
\hline Correlation coeff & -0.04 & 0.6622 & & \\
\hline \multicolumn{5}{|l|}{ Gender } \\
\hline $\begin{array}{l}\text { Male } \\
\text { Female }\end{array}$ & $\begin{array}{l}5.0 \\
5.5\end{array}$ & 0.2843 & & \\
\hline \multicolumn{5}{|c|}{ Histological subtype } \\
\hline $\begin{array}{l}\text { Non-mucinous } \\
\text { Mucinous }\end{array}$ & $\begin{array}{l}5.0 \\
6.0\end{array}$ & 0.2052 & & \\
\hline \multicolumn{5}{|l|}{ Tumour location } \\
\hline $\begin{array}{l}\text { Left } \\
\text { Rectum } \\
\text { Right }\end{array}$ & $\begin{array}{l}4.0 \\
5.0 \\
6.0\end{array}$ & 0.1372 & & \\
\hline \multicolumn{5}{|l|}{ pT } \\
\hline $\begin{array}{l}\text { pT1 + pT2 } \\
\text { pT3 + pT4 }\end{array}$ & $\begin{array}{l}3.5 \\
5.0\end{array}$ & 0.0143 & $1.09(1.014-1.23)$ & 0.644 \\
\hline \multicolumn{5}{|l|}{ pN } \\
\hline $\begin{array}{l}\text { pN0 } \\
\text { pN1-2 }\end{array}$ & $\begin{array}{l}3.5 \\
6.0\end{array}$ & 0.0007 & $1.062(1.014-1.112)$ & 0.672 \\
\hline
\end{tabular}

No. lymph nodes collected

\begin{tabular}{|l|l|l|l|l|}
\hline Correlation coeff & 0.099 & 0.2549 & & \\
\hline \multicolumn{5}{|l|}{ Tumour deposits } \\
\hline 0 & $\begin{array}{l}5.0 \\
6.0\end{array}$ & 0.8695 & & \\
1 or more & & & \\
\hline Metastasis & 4.0 & 0.0013 & $1.05(1.014-1.087)$ & 0.678 \\
\hline CM0 & 8.0 & & & \\
CM1
\end{tabular}

Lymphatic invasion

\begin{tabular}{|l|l|l|l|l|}
\hline L0 & 3.5 & 0.0065 & $1.117(1.025-1.216)$ & 0.667 \\
\hline
\end{tabular}

Venous invasion

\begin{tabular}{|l|l|l|l|l|}
\hline V0 & 5.0 & 0.0318 & $1.054(1.011-1.1)$ & 0.617 \\
V1-2 & 5.5 & & & \\
\hline
\end{tabular}

Perineural invasion $(\mathrm{Pn})$

\begin{tabular}{|l|r|r|l|l|}
\hline 0 & 5.0 & 0.1726 & & \\
1 & 11.0 & & & \\
\hline
\end{tabular}

Tumour grade

\begin{tabular}{|l|l|l|l|l|}
\hline G1-2 & 5.0 & 0.1446 & & \\
G3 & 6.0 & & & \\
\hline
\end{tabular}

Abbreviations: $\quad A U C=$ area under the $\mathrm{ROC}$ curve; $\mathrm{Cl}=$ confidence interval; coeff = coefficient; ITB = intratumoural budding; $O R=$ odds ratio.

tumour buds in the biopsy was significantly associated with a more advanced pT stage $(P=0.0143)$, LN metastasis $(P=0.0007)$, lymphatic vessel invasion $(P=0.0065)$ and venous vessel invasion $(P=0.0318)$ as determined from the surgical specimen.
Additionally, a greater number of buds was observed in patients with distant metastasis (cM1) $(P=0.0013)$.

Next, using the logistic regression equation, a probability scale was derived for predicting the risk of (A) LN metastasis and (B) distant metastasis as a function of the number of tumour buds (Figure 3). The estimates for the intercept and number of buds were -0.1865 and 0.0602 . The greater the number of tumour buds, the greater the probability for local and distant metastasis. For example, a patient with a biopsy containing 10 tumour buds would have a $64 \%$ chance of LN metastasis, whereas the probability increases in a patient with 30 buds or $>50$ buds (i.e., signet ring cell tumour) to $86 \%$ and $95 \%$, respectively. Similarly, a patient with 10 buds in the preoperative biopsy has a low probability of distant metastasis $(30 \%)$ in comparison with a patient with 30 or 50 buds (53\% and $75 \%$, respectively).

\section{DISCUSSION}

The present study demonstrates that the presence of ITB in colon and rectal cancer biopsies (not treated with neoadjuvant therapy) is associated with higher T stage $(P=0.0143)$, lymphatic $(P=0.007)$ and vascular invasion $(P=0.032), \mathrm{LN}(P=0.001)$ and distant metastases $(P=0.001)$. The results underline the diagnostic importance of ITB and are in line with the results from the literature (Morodomi et al, 1989; Lugli et al, 2011; Giger et al, 2012; Rogers et al, 2014). The practical approach to ITB is user-friendly, concise and understandable, three characteristics that are important for the translation of a histopathological biomarker into daily diagnostic practice.

Morodomi et al (1989) previously showed in 1989 the correlation of budding in preoperative biopsies and the presence of LN metastases in 112 rectal cancer patients. These findings were confirmed by Giger et al (2012) analysing preoperative biopsies and the corresponding resections of 72 CRC patients; Rogers et al (2014) additionally underlined the predictive power of ITB for nodal metastases in 89 rectal cancer patients treated with neoadjuvant therapy. Both Giger and Rogers relied on HE-stained sections of the preoperative biopsy and used a semi-quantitative, three-tiered scoring system. In 2011, our research group systematically analysed the presence of budding on 511 whole-tissue sections after pan-cytokeratin staining of CRC which resulted in the concept of stratifying tumour budding into ITB and PTB (Lugli et al, 2011). In fact the presence of tumour budding in preoperative biopsies (ITB) and in corresponding resections (PTB) is strongly and positively correlated suggesting that an overall tumour budding (OTB) count could be useful. We further showed that ITB was an independent prognostic factor. In summary, all these studies highlight the diagnostic, prognostic and predictive potential of ITB.

Strong points of the present study include the selection of the biomarker and the practical approach. Tumour budding is a robust histomorphological marker and declared an independent prognostic factor by many different studies, independently of the selected scoring system (Hase et al, 1993; Okuyama et al, 2002, 2003; Ueno et al, 2002, 2004; Jass et al, 2003; Nakamura et al, 2005; Park et al, 2005; Horkko et al, 2006; Choi et al, 2007; Lugli et al, 2009; Wang et al, 2009). These results are biologically not surprising as tumour buds likely represent an epithelial-mesenchymal transition (EMT)-like process, express proteins of tumour aggressiveness such as matrix-metalloproteinases, nuclear $\beta$ catenin, VEGF, p16 and some potential stem cell markers in association with loss of adhesion molecules such as E-cadherin (Zlobec and Lugli, 2010). Interestingly, tumour budding is completely independent of tumour grade (defined as the percentage of glandular formation), tumour border configuration 
A

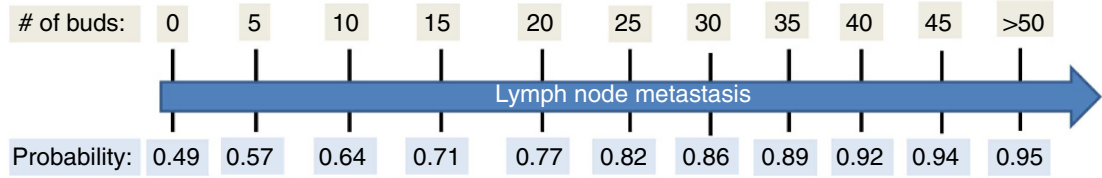

B

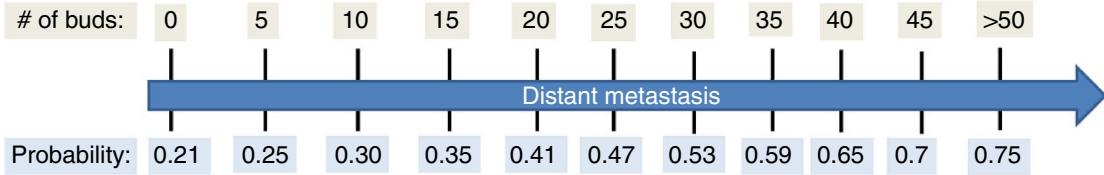

Figure 3. Probability scale. The greater the number of buds is, the greater the risk of metastasis.

and molecular features such as mismatch-repair status, CpG Island Methylator Phenotype (CIMP), KRAS and BRAF mutation status (Zlobec et al, 2012a). The main reason why tumour budding is not completely integrated in the pathology reports is the lack of a standardized scoring system, although, as stated above, the prognostic power seems to be independent of the applied scoring system (Horcic et al, 2013). In this study, we selected a practical approach including two steps: first, the pathologist needs to optimally visualise buds in the preoperative biopsies by using immunohistochemistry (pan-cytokeratin marker), as buds cannot be easily recognised in areas of inflammation. The argument that the use of one single immunostaining is cost-ineffective is not valid as lymphoma, sarcoma and breast cancer diagnostics are based on many immmunohistochemical markers. Second, pathologists are already familiar with counting mitoses and cells in high-power field (HPF) such as in GISTs, lymphomas, sarcomas and breast cancer (BRE score).

On the basis of the actual number of tumour buds, a predictive scale for LN and distant metastasis was constructed. The actual probability for both outcomes could therefore be visualised. Such an approach has the distinct advantage of not relying on a cutoff score for low- or high-grade budding, which will ease the diagnosis of ITB for pathologists as well as the interpretation of the findings for all members of the interdisciplinary team. For the actual number of buds the corresponding probabilities can be determined thereby giving 'relativity' to the findings. Moreover, we show that the inter-observer agreement of ITB is strong using the hot-spot method, again underlining the advantage of this approach.

There are at least three scenarios where ITB detected from preoperative biopsies may have clinical relevance. The first is in patients with clinical stage II rectal cancers. Although most patients with stage II tumours are offered a neoadjuvant therapy, the presence of ITB in the pretreatment biopsy is an estimator of tumour behaviour that may help to stratify patients into highand low-risk groups. The second may be in the preoperative management of patients with clinical stage II colon cancers. Although these cancers are not typically treated in the neoadjuvant setting, evidence from the recent pilot phase of the FOXTROT trial for locally advanced colon cancer patients shows the feasibility and possible survival benefit of preoperative chemotherapy (Foxtrot Collaborative G, 2012). Intratumoural budding may help to identify patients with stage II cancers who are at high-risk for nodal metastasis, who may be candidates for neoadjuvant chemotherapy. The third is in patients with submucosally invasive (T1) CRCs after endoscopic resection that may be at high-risk of LN positivity, and thus would benefit from consequent surgical resection. In this context, at least a dozen studies (reviewed in (Zlobec et al, 2012b)) have shown that tumour budding detected in the surgical resection specimen predicts the future development of LN positivity in these early $\mathrm{T} 1$ cancers.
One weakness of our study is the sample size (especially the number of rectal cancer cases). However, in addition to the already published results in the literature and considering the fact that also in colon cancer ITB will have an important role in the preoperative management, the weakness may be considered marginal. In fact, this appears to be the largest study to date to evaluate ITB in preoperative biopsies.

In conclusion, our results highlight ITB as a histomorphological parameter in preoperative biopsies of colon and rectal cancer patients. This study together with previous works has laid a solid retrospective foundation for future large prospective studies.

\section{ACKNOWLEDGEMENTS}

We thank Mary Economou and Caroline Hammer from the Translational Research Unit, Institute of Pathology, University of Bern for excellent technical support. This study was supported by the Bernese Cancer League.

\section{CONFLICT OF INTEREST}

The authors declare no conflict of interest.

\section{REFERENCES}

Bosman FT, Carneiro F, Hruban RH (eds) (2010) WHO Classification of Tumours of the Digestive System. WHO; International Agency for Research on Cancer (IARC).

Choi HJ, Park KJ, Shin JS, Roh MS, Kwon HC, Lee HS (2007) Tumor budding as a prognostic marker in stage-III rectal carcinoma. Int J Colorectal Dis 22(8): 863-868.

Foxtrot Collaborative G (2012) Feasibility of preoperative chemotherapy for locally advanced, operable colon cancer: the pilot phase of a randomised controlled trial. Lancet Oncol 13(11): 1152-1160.

Giger O, Comtesse S, Lugli A, Zlobec I, Kurrer M (2012) Intra-tumoral budding (ITB) in pre-operative biopsy specimens predicts lymph node and distant metastasis in patients with colorectal cancer. Mod Pathol 25(7): 1048-1053.

Hase K, Shatney C, Johnson D, Trollope M, Vierra M (1993) Prognostic value of tumor 'budding' in patients with colorectal cancer. Dis Colon Rectum 36(7): 627-635.

Horcic M, Koelzer VH, Karamitopoulou E, Terracciano L, Puppa G, Zlobec I, Lugli A (2013) Tumor budding score based on 10 high-power fields is a promising basis for a standardized prognostic scoring system in stage II colorectal cancer. Hum Pathol 44(5): 697-705.

Horkko TT, Klintrup K, Makinen JM, Napankangas JB, Tuominen HJ, Makela J, Karttunen TJ, Makinen MJ (2006) Budding invasive margin and prognosis in colorectal cancer-no direct association with beta-catenin expression. Eur J Cancer 42(7): 964-971. 
Jass JR, Barker M, Fraser L, Walsh MD, Whitehall VL, Gabrielli B, Young J, Leggett BA (2003) APC mutation and tumour budding in colorectal cancer. J Clin Pathol 56(1): 69-73.

Lugli A, Karamitopoulou E, Panayiotides I, Karakitsos P, Rallis G, Peros G, Iezzi G, Spagnoli G, Bihl M, Terracciano L, Zlobec I (2009) CD8 + lymphocytes/tumour-budding index: an independent prognostic factor representing a 'pro-/anti-tumour' approach to tumour host interaction in colorectal cancer. Br J Cancer 101(8): 1382-1392.

Lugli A, Vlajnic T, Giger O, Karamitopoulou E, Patsouris ES, Peros G, Terracciano LM, Zlobec I (2011) Intratumoral budding as a potential parameter of tumor progression in mismatch repair-proficient and mismatch repair-deficient colorectal cancer patients. Hum Pathol 42(12): 1833-1840.

Morodomi T, Isomoto H, Shirouzu K, Kakegawa K, Irie K, Morimatsu M (1989) An index for estimating the probability of lymph node metastasis in rectal cancers. Lymph node metastasis and the histopathology of actively invasive regions of cancer. Cancer 63(3): 539-543.

Nakamura T, Mitomi H, Kikuchi S, Ohtani Y, Sato K (2005) Evaluation of the usefulness of tumor budding on the prediction of metastasis to the lung and liver after curative excision of colorectal cancer. Hepatogastroenterology 52(65): 1432-1435.

Okuyama T, Nakamura T, Yamaguchi M (2003) Budding is useful to select high-risk patients in stage II well-differentiated or moderately differentiated colon adenocarcinoma. Dis Colon Rectum 46(10): 1400-1406.

Okuyama T, Oya M, Ishikawa H (2002) Budding as a risk factor for lymph node metastasis in pT1 or pT2 well-differentiated colorectal adenocarcinoma. Dis Colon Rectum 45(5): 628-634.

Park KJ, Choi HJ, Roh MS, Kwon HC, Kim C (2005) Intensity of tumor budding and its prognostic implications in invasive colon carcinoma. Dis Colon Rectum 48(8): 1597-1602.

Prall F, Nizze H, Barten M (2005) Tumour budding as prognostic factor in stage I/II colorectal carcinoma. Histopathology 47(1): 17-24.

Rogers AC, Gibbons D, Hanly AM, Hyland JMP, O'Connell PR, Winter DC, Sheahan K (2014) Prognostic significance of tumor budding in rectal cancer biopsies before neoadjuvant therapy. Mod Pathol 27(1): 156-162.

Schmoll HJ, Van Cutsem E, Stein A, Valentini V, Glimelius B, Haustermans K, Nordlinger B, van de Velde CJ, Balmana J, Regula J, Nagtegaal ID,
Beets-Tan RG, Arnold D, Ciardiello F, Hoff P, Kerr D, Kohne CH, Labianca R, Price T, Scheithauer W, Sobrero A, Tabernero J, Aderka D, Barroso S, Bodoky G, Douillard JY, El Ghazaly H, Gallardo J, Garin A, Glynne-Jones R, Jordan K, Meshcheryakov A, Papamichail D,

Pfeiffer P, Souglakos I, Turhal S, Cervantes A (2012) ESMO Consensus Guidelines for management of patients with colon and rectal cancer. A personalized approach to clinical decision making. Ann Oncol 23(10): $2479-2516$

Tanaka M, Hashiguchi Y, Ueno H, Hase K, Mochizuki H (2003) Tumor budding at the invasive margin can predict patients at high risk of recurrence after curative surgery for stage II, T3 colon cancer. Dis Colon Rectum 46(8): 1054-1059.

Ueno H, Murphy J, Jass JR, Mochizuki H, Talbot IC (2002) Tumour 'budding' as an index to estimate the potential of aggressiveness in rectal cancer. Histopathology 40(2): 127-132.

Ueno H, Price AB, Wilkinson KH, Jass JR, Mochizuki H, Talbot IC (2004) A new prognostic staging system for rectal cancer. Ann Surg 240(5): 832-839.

Wang LM, Kevans D, Mulcahy H, O'Sullivan J, Fennelly D, Hyland J, O'Donoghue D, Sheahan K (2009) Tumor budding is a strong and reproducible prognostic marker in T3N0 colorectal cancer. Am J Surg Pathol 33(1): 134-141.

Zlobec I, Bihl MP, Foerster A, Rufle A, Lugli A (2012a) The impact of $\mathrm{CpG}$ island methylator phenotype and microsatellite instability on tumour budding in colorectal cancer. Histopathology 61(5): 777-787.

Zlobec I, Borner M, Lugli A, Inderbitzin D (2012b) Role of intra- and peritumoral budding in the interdisciplinary management of rectal cancer patients. Int J Surg Oncol 2012: 795945.

Zlobec I, Lugli A (2010) Epithelial mesenchymal transition and tumor budding in aggressive colorectal cancer: tumor budding as oncotarget. Oncotarget 1(7): 651-661.

This work is published under the standard license to publish agreement. After 12 months the work will become freely available and the license terms will switch to a Creative Commons AttributionNonCommercial-Share Alike 3.0 Unported License. 